\title{
XXXIV. On rays of electric force
}

\section{Prof. Dr. H. Hertz}

To cite this article: Prof. Dr. H. Hertz (1889) XXXIV. On rays of electric force, Philosophical Magazine Series 5, 27:167, 289-298, DOI: 10.1080/14786448908628357

To link to this article: http://dx.doi.org/10.1080/14786448908628357

$$
\text { 册 Published online: } 29 \text { Apr } 2009 .
$$

Submit your article to this journal 준

LII Article views: 5

Q View related articles $\asymp$ 


\title{
LONDON, EDINBURGH, AND DUBLIN
}

\section{PHILOSOPHICAL MAGAZINE}

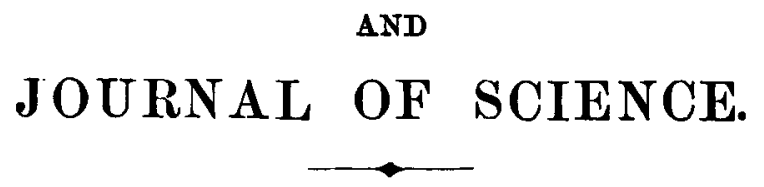

[FIFTH SERIES.]

$A P R I L 1889$.

XXXIV. On Rays of Electric Force.

By Prof. Dr. H. Hertz*.

\begin{abstract}
A FTER I had succeeded in showing that an electric oscilA lation could give rise to a wave capable of radiating into space, I at once made the attempt to intensify this effect and to make it sensible at greater distances by placing the exciting conductor in the focus of a large parabolic mirror. These attempts did not succeed, and I convinced myself that the failure was the necessary result of the wrong proportion existing between the length of the waves employed, viz. 4 to 5 metres, and the dimensions of the mirror in the most favourable case possible. I have lately observed that the experiments described by me can quite well be performed with oscillations more than ten times as rapid, and with waves more than ten times as short as those at first discovered. I have therefore returned to the use of concave mirrors, and have attained better results than I had ventured to expect. I have succeeded in producing distinct rays of electric force, and in performing with them the elementary experiments which one is accustomed to perform with light and with radiant heat. An account is here given of these experiments.
\end{abstract}

\section{The Apparatus.}

The method of producing short waves is the same as that by which the longer waves were excited. The primary con-

* Translated from the Sitzungsb. der Akad. der Wiss. Berlin, Dec. 13, 1888.

Phil. Mag. S. 5. Vol. 27. No. 167. April 1889. U 
ductor employed may be most simply described as follows :Imagine a cylindrical brass body, of 3 centim. diameter and 26 centim. length, interrupted in the middle of its length by a spark-space of which the poles on each side consist of spherical surfaces of 2 centim. radius. The length of the conductor is nearly equal to half the wave-length of the corresponding oscillations in straight wires; we can thus form at once an approximate estimate of the period of oscillation. It is essential that the pole-surfaces of the spark-interval should be frequently polished, and during the experiments carefully guarded against illumination by simultaneous lateral discharges, otherwise the oscillations are not obtained.

The aspect and the sound of the spark both give notice whether the spark-interval is in a satisfactory condition. The discharge is conducted to the two halves of the conductor by means of two thick wires covered with gutta-percha, which terminate near the spark-interval on the two sides. As inductorium I found it advantageous to employ, instead of the large Rühmkorff's apparatus, a small coil by Keiser and Schmidt, capable of giving sparks of, at most, 4.5 centim. length. It was worked by three accumulators, and gave between the spherical surfaces of the primary conductor sparks of from 1 to 2 centim. long. The spark-interval was then adjusted for the experiments to a length of about 3 millim.

The evidence of the electric forces in space was obtained here also by means of the small sparks which they produced in a secondary conductor. As before, I made use partly of a movable circle, which had an oscillation-period nearly equal to that of the primary conductor. This had now a diameter of 7.5 centim., and was constructed of copper wire 1 millim. thick. The one end of the wire carried a polished brass ball of some millimetres diameter ; the other end was pointed, and was adjusted by means of a fine screw, insulated from the wire, to an extremely small distance from the brass ball. It will easily be understood that we have to do only with little sparks of a few hundredths of a millimetre in length, and a little practice enables one to judge better from the brightness of the sparks than from their length.

The circular conductor gives only a differential effect, and is not suitable for placing in the focal line of a concave mirror; it was therefore combined with another secondary conductor of the following form:-Two straight pieces of wire, 50 centim. long and 5 millim. diameter, were so arranged in the same straight line that the opposing ends were 5 centim. apart. From these ends two wires, 15 centim. 
long and 1 millim. thick, led, parallel to each other and at right angles to the first-mentioned wires, to a spark-interval similarly arranged to that of the circular conductor. In this conductor no attention was paid to resonance, which is here not very perceptible. It would have been more simple to place the spark-interval directly in the middle of the straight wire ; but the spark-interval could not then have been manipulated and observed in the focus of the concave mirror without the observer obstructing the aperture of the mirror. For this reason the arrangement described was preferred as more advantageous.

\section{The Production of the Ray.}

If the primary vibration be now set up in a large free space, the circular conductor in its neighbourhood permits the observation, on a smaller scale, of all the phenomena which I had previously observed and described in the neighbourhood of a larger oscillation*.

The greaiest distance at which sparks could be recognized in the secondary conductor was 1.5 metre, or as much as 2 metres with a favourable condition of the primary sparkinterval.

The effect is increased on either side if a plane conducting surface is adjusted on the opposite side, parallel to the oscillation and at a suitable distance. If the distance is chosen either very small or somewhat greater than 30 centim. the surface produces a prejudicial effect; it produces a strong reinforcement at distances between 8 and 15 centim., a feeble reinforcement at 45 centim., and is without effect at greater distances. We have previously remarked upon this phenomenon, and we conclude from it that the wave corresponding to the primary oscillation has, in air, a half wave-length of about 30 centim. We might expect a still greater reinforcement by replacing the plane surface by a concave mirror of the form of a parabolic cylinder, in the focus of which the longer axis of the primary oscillation falls. If the mirror is properly to concentrate the action at a distance, it is advantageous to have its focal length as small as possible. But if the direct wave is not immediately to quench the action of the reflected wave, the focal length must not be much less than a quarter of a wave-length. I chose therefore a focal length of $12 \frac{1}{2}$ centim., and constructed the mirror by bending a zinc plate, 2 metres long, 2 metres broad, and $\frac{1}{2}$ millim. thick, round a wooden frame of the right curvature into the desired

* H. Hertz, Wiedemann's Annalen, xxxiv. pp. 155, 551, 609. 
form. The height of the mirror thus became 2 metres, the diameter of its aperture 1.2 metre, and its depth 0.7 metre. The primary oscillation was adjusted in the centre of its focal line. The wires by which the discharge was conducted were allowed to traverse the mirror ; the inductorium and the accumulators were placed behind the mirror, and produced no disturbance. If, now, we examine the neighbourhood of the vibration by means of our conductors, we find no action behind the mirror and at one side in general ; but in the direction of the optic axis of the mirror the sparks are perceptible to a distance of 5 or 6 metres. By placing a plane conducting surface at right angles to the advancing wave the sparks were perceptible near it to a much greater distance-as much as 9 or 10 metres.

The waves reflected by the conducting surface strengthen the direct waves at certain points. At other points, again, the two waves weaken each other. With the straight conductor we can recognize very distinct maxima and minima in front of the plane conductor, and with the circular conductor the interference phenomena characteristic of stationary waves, which I have previously described. I was able to disinguish four nodes which fell on the wall, at 33 , at 65 , and at 98 centim. distance from it respectively. Hence we have 33 centim. as a close approximation to the half wave-length of the waves employed, and an oscillation-period of $1 \cdot 1$ thousand millionths of a second, assuming the velocity of light for the velocity of radiation. In wires the oscillation had a wave-length of 29 centim. It seems, then, that also with these short waves the velocity is somewhat less in wires than the velocity in space; but the ratio of the two velocities comes very near to the theoretical value of 1 , and does not deviate from it so much as our experiments with longer waves made probable. This remarkable phenomenon still requires explanation. Since these phenomena are manifested especially in the neighbourhood of the optic axis of the mirror, we designate the result as an electric ray issuing from the concave mirror.

I now made a second concave mirror exactly similar to the first, and placed the straight secondary conductor in it, so that the two 50 centim. long wires fell in the focal line, but the two wires leading to the spark-interval traversed the wall of the mirror (being insulated from it) by the shortest route. The spark-interval was thus close behind the mirror, and the observer could adjust and observe it without interrupting the course of the waves. I expected that on intercepting the ray by this arrangement I should be able to recognize it at still greater distances, and I found that I was not mistaken. In 
the space at my disposal I could recognize the sparks from one end to the other. The greatest distance to which-by making use of an open door-I could trace the ray was 16 metres ; but the results of the experiments on reflexion, to be described immediately, leave no doubt that in open spaces sparks could be obtained up to at least 20 metres. For the remaining experiments so great a distance is not necessary, and it is more convenient if the secondary spark is not too weak ; a distance of 6-10 metres is the most advantageous for most experiments. We will now describe the simple experiments which may be made with the ray without difficulty. When the opposite is not expressly remarked, the focal lines of both mirrors are to be supposed vertical.

\section{Rectilinear Radiation.}

If a screen of zinc plate 2 metres high and 1 metre broad be placed in the straight line joining the two mirrors at right angles to the direction of the ray, the secondury sparks completely disappear. A screen of tin-foil or of gilt paper gives an equally perfect shadow. A frame placed across the ray leaves the secondary spark-interval dark whenever it intercepts the ray, and permits the sparks to appear whenever it allows the ray to pass. Insulators do not intercept the ray-it passes through a screen of wood or through a wooden door, and it is not without surprise that we see the sparks produced inside a closed room. If two conducting screens, each 2 metres high and 1 metre broad, are placed diametrically right and left near the ray and at right angles to its direction, they do not influence the secondary sparks at all, so long as the breadth of the slit which they leave between them is not smaller than the aperture of the mirror, that is 1.2 metres. If the slit is made narrower than this, the sparks diminish and disappear when the breadth of the slit becomes less than 0.5 metre. If the breadth of the slit be left 1.2 metres, but the screens be moved sideways out of the direct line joining the two mirrors, the sparks also disappear. If the producing mirror be turned right or left, through about $10^{\circ}$ out of the correct position, the sparks become weaker, and when turned through $15^{\circ}$ the sparks disappear.

The ray has no geometrically sharp limit or shadow, and it is easy to obtain phenomena due to refraction, but I have not yet succeeded in observing maxima and minima at the edge of a shadow.

\section{Polarization.}

The mode of production of our ray leaves no doubt that it 
consists in transverse vibrations, and can be "plane-polarized" in the optical sense. This fact, however, may also be shown by experiment. If the receiving mirror be rotated round the ray as axis until its focal line, and consequently also the secondary conductor, have attained the horizontal position, the secondary sparks disappear gradually, and no sparks are to be obtained when the two focal lines are at right angles, even if the two mirrors are made to approach each other closely. The two mirrors behave like the polarizer and analyzer of a polarizing apparatus. I had made an octagonal frame of wood 2 metres high and 2 metres broad, and wound copper of 1 millim. thick wire over this, so that the wires were all parallel and 3 centim. from each other. If now the two mirrors were arranged with parallel focal lines, and the wire screen was moved about in the ray at right angles to its direction so that the wires were at right angles to the plane of the focal lines, the screen produced practically no effect upon the secondary sparks; but if the screen was placed so that its wires were parallel to the plane of the focal lines, the ray disappeared altogether. Thus, in regard to the transmitted energy, the screen behaves to the ray exactly like a tourmaline plate to a plane-polarized ray. The focal line of the receiving mirror was then placed horizontal, in which position, as already mentioned, no sparks were obtained, nor were sparks produced by the introduction of the screen into the ray, so long as its wires were either vertical or horizontal. But if the wire frame was placed in either of the two possible positions so that its wires made an angle of $45^{\circ}$ with the horizontal line, the introduction of the screen caused the production of sparks. Evidently the screen produces the resolution of the vibrations into two components, and transmits only that vibration which takes place at right angles to the direction of the wires. This component is inclined at an angle of $45^{\circ}$ to the focal line of the second mirror, and when once more resolved by the mirror produces the action upon the secondary conductor. The phenomenon is exactly similar to the brightening produced in the dark field of two crossed nicols by a tourmaline plate introduced in the proper position.

The following remarks upon the subject of polarization may be permitted. With the means employed in the present investigation we are only able to recognize electric force. Its vibrations undoubtedly take place, for a vertical position of the primary vibration, in the vertical plane passing through the ray, and are wanting in the horizontal plane. But from the phenomena we observe with slowly changing currents we 
cannot doubt that the electric vibrations are accompanied by vibrations of magnetic force, which take place in the horizontal plane and become zero in the vertical plane. The question in which of the two planes the vibrations of our ray take place does not, then, admit of an answer without data to determine whether the question is of electric or of magnetic vibrations. That the failure to decide an old optical dispute is explained by this consideration was first clearly pointed out by Herr Kolaček*.

\section{Reflexion.}

We have already proved the reflexion of the wave at a conducting surface by the interference' of the reflected and direct waves, and have made use of it in the construction of our concave mirrors. But it is now possible for us to separate the two wave-systems. I placed the two concave mirrors in an open space side by side, so that their apertures faced the same way, and that their axes converged upon a point about 3 metres off. The spark-space of the receiving mirror of course remained dark. Now I placed a vertical plane wall of thin zinc plate 2 metres high and 2 metres broad at the intersection of the axes, so as to be equally inclined to them both. I then obtained a long stream of sparks resulting from the reflected rays. The stream of sparks ceased as soon as the wall was turned about a vertical axis either way through about $15^{\circ}$ from the correct position ; hence the reflexion is regular, and not diffuse. If the wall was removed from the mirrors, their axes being made to converge always upon the wall, the sparks diminished very slowly. I was still able to recognize sparks when the wall was distant 10 metres from the mirrors ; the waves had then to traverse a path of 20 metres. This arrangement might be employed with advantage if it were desired to compare the velocity of radiation in the air with other slower velocities of propagation, e.g. that by means of a cord.

In order to obtain the reflexion of the ray at incidences other than normal, I arranged the ray parallel to a wall in which there was a door, and in the neighbouring room to which the door led I placed the receiving mirror so that its optic axis traversed the centre of the door and cut the direction of the ray at right angles. If, now, the plane conducting screen was placed vertically at the point of intersection so as to make angles of $45^{\circ}$ both with the ray and with the axes of the receiving mirror, a stream of sparks was produced in the

* Wiedemann's Annalen, xxxiv. p. 676. 
secondary conductor which also was not interfered with by the closing of the door. If the reflecting screen was turned through about $10^{\circ}$ from the right position, the sparks ceased. The reflexion is therefore regular, and the angles of incidence and reflexion are equal. That the action was transmitted from the source to the plane mirror and from there to the secondary conductor could álso be shown by interposing shadow-giving screens at different points of this path, when the secondary sparks at once ceased, whilst the screen could be placed anywhere else in the room without effect. With the aid of the circular secondary conductor it is possible to determine the position of the wave-surface in the ray; this was at right angles to the ray, both before and after reflexion, so that in the reflexion it suffers a deviation of $90^{\circ}$.

So far the focal lines of the concave mirrors have been vertical, and the plane of vibration consequently at right angles to the plane of incidence. In order to produce reflexion with the vibrations in the plane of incidence, I adjusted the focal lines of both concave mirrors in the horizontal plane. I observed the same phenomena as in the former case, and was moreover not able to perceive a difference in the intensity of the reflected rays in the two cases. If, on the other hand, the focal line of the one mirror is vertical and that of the other horizontal, no secondary sparks are to be perceived. The inclination of the plane of vibration to the plane of incidence is therefore not altered by reflexion so long as this inclination has one of the values mentioned; but this statement cannot be taken as generally true. It may even be considered as open to doubt whether generally the ray after reflexion is plane-polarized. The interferences which the intersecting systems of waves produce before the mirror and which, as I observed, give rise to characteristic phenomena in the circular conductor, may possibly lead to conclusions in the current problems of the optician as to change of phase and amplitude upon reflexion.

I will mention one other experiment on reflexion by an isotropic surface. The two curved mirrors were again placed side by side as in the experiment on reflexion first described, and opposite to them as a reflecting wall the screen, constructed of parallel copper wires, was placed.

It was seen that the secondary spark-interval remained dark when the wires intersected the direction of the vibrations at right angles, but became brighter as soon as the wires coincided with the direction of vibration. The analogy between our surface conducting in one direction and the tourmaline plate is therefore limited to the transmitted portion of the ray. The 
part not transmitted is absorbed by the tourmaline plate but reflected by our surface. If in the last experiment the focal lines of the two mirrors are crossed, we can obtain no sparks in the secondary conductor by reflexion at an isotropic surface; but I convinced myself that the experiment succeeds with reflexion at an anisotropic wire screen, if so placed that its wires are inclined at an angle of $45^{\circ}$ to both focal lines. The experiment is easily understood from what has been said.

\section{Refraction.}

In order to try if a bending of the ray could be observed upon its passing from air into another insulating medium, I had constructed a large prism of so-called hard pitch, an asphalt-like material. The base was an equilateral triangle 1.2 metre in the side, and with a refracting angle of about $30^{\circ}$. The height of the prism with its refracting edge placed vertical was about 1.5 metre.

But since the prism weighed about 12 cwt. and altogether was inconveniently heavy, it was constructed in three pieces, each about 0.5 metres high, placed one on the other. The mass was cast in wooden boxes, which, since they would have no prejudicial effect, were left round the mass. The prism was placed in a support at such a height that the centre of its refracting edge was at the same height as the primary and secondary spark-intervals. After I had convinced myself that refraction did occur, and had formed an opinion as to its amount, I arranged the experiment as follows:-The producing mirror was placed at a distance of 2.6 metres from the prism, turned towards the one refracting surface, so that the axis of the ray passed through the centre of gravity of the prism and intersected the refracting surface at an angle of $65^{\circ}$. Two conducting-screens were placed near the refracting edge of the prism and near the opposite side, which cut off from the ray every other part than that through the prism. On the side of the emerging ray a circle was traced upon the floor of 2.5 metres radius, with the centre of gravity of the prism as centre. The receiving mirror was moved about upon this, so that its aperture was always directed towards the centre of the circle. If the mirror were placed in the line of the incident ray, no sparks were to be obtained in it; in this direction the prism threw a perfect shadow. Sparks, however, appeared when the mirror was moved towards the second surface of the prism, and first when the angular displacement was about $11^{\circ}$. The sparks increased in intensity up to a deviation of about $22^{\circ}$ and then decreased again, becoming imperceptible at about $34^{\circ}$. If the mirror 
was adjusted in the position where the strongest effect was observed, and withdrawn from the prism along a radius of the circle, the sparks could be recognized up to a distance of 5 or 6 metres. A screen placed in front of or behind the prism always quenched the sparks; a proof that the action occurred in fact through the prism, and did not reach the secondary conductor by any other part. The experiments were repeated after placing the focal lines horizontal, but without altering the position of the prism. No alteration in the phenomena produced was observed. A refracting angle of $30^{\circ}$ and a deviation of $22^{\circ}$ in the neighbourhood of minimum deviation corresponds to a refractive index of $1 \cdot 69$. The refractive index for light is given for pitch-like substances between 1.5 and 1·6. The uncertainty of our determination and the impurity of the material employed does not permit of our assigning greater importance to the magnitude or signification of this difference.

We have represented the phenomena investigated by us as rays of electric force. We may in conclusion perhaps regard them as light-rays of very great wave-length. To me at least the experiments described seem eminently fitted to remove all doubt as to the identity of light, radiant heat, and electrodynamic wave-motion. I believe that we shall now with more confidence avail ourselves of the advantages which the assumption of this identity offers, both in the domains of optics and of electricity.

$\mathrm{XXXV.} \mathrm{On} \mathrm{the} \mathrm{Limit} \mathrm{to} \mathrm{Interference} \mathrm{when} \mathrm{Light} \mathrm{is} \mathrm{radiated}$ from Moving Molecules. By Lord RAYLEIGH*.

工 N a recent number of Wiedemann's Annalen, Ebert $\dagger$ discusses the application of Doppler's principle to the radiation from the moving molecules of an incandescent gas $\ddagger$, and arrives at the conclusion that the widths of the spectral lines, as calculated upon the basis of the principle, are much greater than is consistent with experiments upon interference with a large relaive retardation. This is a matter of no small importance. Unless the discrepancy can be explained, the dynamical theory of gases would, it appears to me, have received a heavy blow, from which it could with difficulty recover. If it be true that a gas consists of molecules in irregular motion,

* Communicated by the Author.

† Wied. Ann. xxxvi. p. 466 (1889).

Lippich, Pogg. Ann. cxxxix. p. 465 (1870). Rayleigh, 'Nature,' viii. p. 474 (1873). 